\title{
Hematopoietic cell transplantation and cellular therapy survey of the EBMT: monitoring of activities and trends over 30 years
}

\author{
Jakob R. Passweg ${ }^{1}$ Helen Baldomero $\mathbb{1}^{1} \cdot$ Christian Chabannon $\mathbb{1}^{2} \cdot$ Grzegorz W. Basak $\mathbb{D}^{3} \cdot$

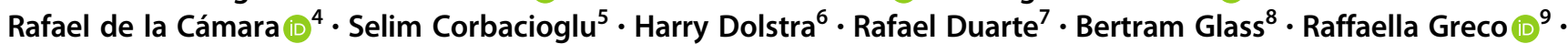 \\ Arjan C. Lankester ${ }^{10} \cdot$ Mohamad Mohty $^{11} \cdot$ Régis Peffault de Latour ${ }^{12} \cdot$ John A. Snowden $^{13}$. \\ Ibrahim Yakoub-Agha $\mathbb{C}^{14}$. Nicolaus Kröger ${ }^{15} \cdot$ for the European Society for Blood and Marrow Transplantation \\ (EBMT)
}

Received: 18 December 2020 / Revised: 12 January 2021 / Accepted: 25 January 2021 / Published online: 23 February 2021

(c) The Author(s) 2021. This article is published with open access

\begin{abstract}
Numbers of Hematopoietic cell transplantation (HCT) in Europe and collaborating countries continues to rise with 48,512 HCT in 43,581 patients, comprising of 19,798 (41\%) allogeneic and 28,714 (59\%) autologous, reported by 700 centers in 51 countries during 2019. Main indications were myeloid malignancies 10,764 (25\%), lymphoid malignancies 27,895 (64\%), and nonmalignant disorders 3173 (7\%). A marked growth in CAR-T cellular therapies from 151 in 2017 to 1134 patients in 2019 is observed. This year's analyses focus on changes over 30 years. Since the first survey in 1990 where 143 centers reported $4234 \mathrm{HCT}$, the number has increased to 700 centers and 48,512 HCT. Transplants were reported in 20 countries in 1990, and 51, 30 years later. More than 800,000 HCT in 715,000 patients were reported overall. Next to the massive expansion of HCT technology, most notable developments include the success of unrelated donor and haploidentical HCT, an increase followed by decrease in the number of cord blood transplants, use of reduced intensity HCT in older patients, and the phenomenal rise in cellular therapy. This annual report of the European Society for Blood and Marrow Transplantation (EBMT) reflects current activity and highlights important trends vital for health care planning.
\end{abstract}

\section{Introduction}

The European Society for Blood and Marrow Transplantation (EBMT) published for the first time in 1990 [1] a survey describing activity in hematopoietic stem cell transplant centers in Europe. Since then, this survey was published annually and herewith will be the 30th anniversary edition. The survey includes over 700,000 patients with over 800,000 transplants. Initially the survey was designed in the form of a single page spreadsheet for ease of

A full list of members and their affiliations appears in the Supplementary Information.

Supplementary information The online version contains supplementary material available at https://doi.org/10.1038/s41409021-01227-8.

Jakob R. Passweg

jakob.passweg@usb.ch

Extended author information available on the last page of the article reporting and has remained in this format ever since, although many additional features had been added, such as refined disease classification, information on conditioning intensity, pediatric activity, cell source, and cellular therapy.

Hematopoietic cell transplantation (HCT) is an established procedure for many acquired or inherited disorders of the hematopoietic system, benign or neoplastic, including those of the immune system, and as enzyme replacement in metabolic disorders [2-4]. The activity survey of the EBMT, describing the status of HCT, has become an instrument with which to observe trends and monitor changes in HCT technology in Europe and neighboring countries [5-14]. The survey, using a standardized structure, captures the numbers of HCT from highly committed participating centers, stratified by indication, donor type, and stem cell source. In the last few years, the survey has also included information on cellular therapies with hematopoietic cells for uses other than to replace the hematopoietic system [15-28, https://www.ema.europa.eu/en/ documents/scientific-guideline/qualification-opinion-cellula r-therapy-module-european-society-blood-marrow-transpla 
ntation-ebmt_en.pdf]. The analysis of the survey data since 1990 shows a continued and constant increase in the annual numbers of HCT and transplant rates for both allogeneic and autologous HCT. This report, based on the 2019 survey data, shows recent trends, changes in indications, and use in Europe and collaborating countries and summarizes the last 30 years.

\section{Patients and methods}

\section{Data collection and validation}

We invited participating centers to report their data for 2019 using the activity survey as shown in Table 1 . The survey allows the reporting of additional information on the numbers of subsequent transplants performed due to relapse, rejection, or those that are part of a planned sequential protocol. Information on the numbers of patients receiving unmanipulated donor lymphocyte infusions (DLIs), nonmyeloablative or reduced intensity HCT, and the numbers of pediatric HCT is also collected.

In addition, centers report information on different types of cellular therapies qualifying as advanced therapy medicinal products (ATMP) since they result from substantial manipulations of the collected cells, whether manufactured by industry centrally, or locally by an academic institution.

Quality control measures included several independent systems: confirmation of validity of the entered data by the reporting center, selective comparison of the survey data with MED-A data sets in the EBMT Registry database and crosschecking with National Registries.

\section{Centers}

Since 1990, a directory of HCT centers consisting of both members of the EBMT and non members, in both European and collaborating non-European countries has been accrued. The directory is updated annually according to the centers current activity. In 2019, 730 centers from 53 countries were contacted (42 European and 11 collaborating countries); of which 700 centers responded. This corresponds to a $96 \%$ return rate and includes $82 \%$ of EBMT members. Thirty active centers failed to report in 2019. Reporting centers are listed in the Supplementary Online Appendix in alphabetical order, by country, city, and EBMT center code, with their reported numbers of first and total HCT, and of first allogeneic and autologous HCT. The WHO regional office definitions were used to classify countries as European or non-European. Ten collaborating non-European countries participated in the 2019 EBMT survey: Algeria, Iran, Iraq, Jordan, Lebanon, Nigeria, Saudi Arabia, South Africa, Syria, and Tunisia. Their data, 2590
HCT in 2502 patients, from 31 actively transplanting centers make up $5.3 \%$ of the total data set and are included in all analyses.

\section{Patient and transplant numbers}

Wherever appropriate, patient numbers corresponding to the number of patients receiving a first transplant in 2019, and transplant numbers reflecting the total number of transplants performed are listed. The term sibling donor includes HLA identical siblings and twins but not siblings with HLA mismatches. Unrelated donor transplants include HCT from matched or mismatched unrelated donors with peripheral blood and bone marrow as a stem cell source but not cord blood HCT. Haploidentical transplants are being described as any family member with 2 or more (but not more than 5) loci mismatches within the loci HLA-A, -B, $-\mathrm{C}$, -DRB1, and -DQB1 in GvH and/or HvG direction. Other family member donors are those related donors that are mismatched to a lesser degree than a full haplotype. For the purpose of analysis we add the small number of "other family donor" to haploidentical donor HCT. Additional non-first transplants may include multiple transplants defined as subsequent transplants within a planned double or triple autologous or allogeneic transplant protocol, and re-transplants (autologous or allogeneic) defined as unplanned HCT for rejection or relapse after a previous HCT.

\section{Hematopoietic advanced cellular therapies other than hematopoietic cell transplantation}

Centers were requested to report all patients receiving cellular therapies other than HCT in 2019. Hematopoietic advanced cellular therapies were defined as infusion of cells undergoing substantial manipulation after collection, either selection and/or expansion, or genetic modification and thus qualify as investigational or approved ATMPs according to Regulation (EC) $\mathrm{N}^{\circ}$ 1394/2007. In this context, "substantial" should be understood as referring to the definition included in the Regulation and subsequent regulatory documents and may not reflect the workload assumed by cell processing facilities working in conjunction with clinical programs. Depending on their nature and indications, hematopoietic cellular therapies may be designed to replace or to complement HCT. Administration of non-substantially manipulated hematopoietic cells, such as transplantation of CD34+ selected hematopoietic stem cells is counted as HCT and not as cellular therapy [15]. Similarly, unmanipulated lymphocyte infusions post-HCT are counted as DLIs and not cellular therapy. Hematopoietic cellular therapies include immune effector cells as defined in FACTJACIE standards for Hematopoietic Cellular Therapy: 


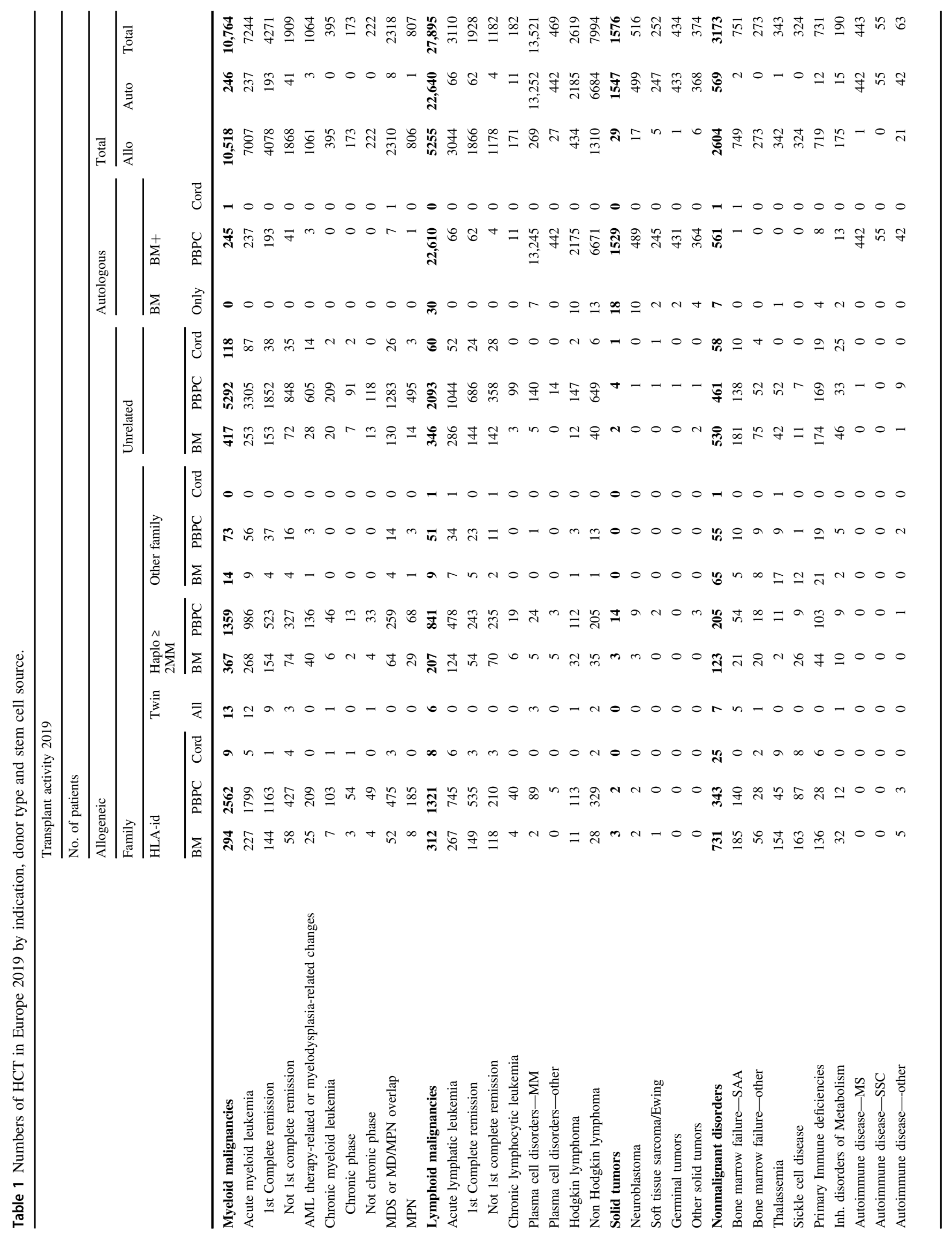




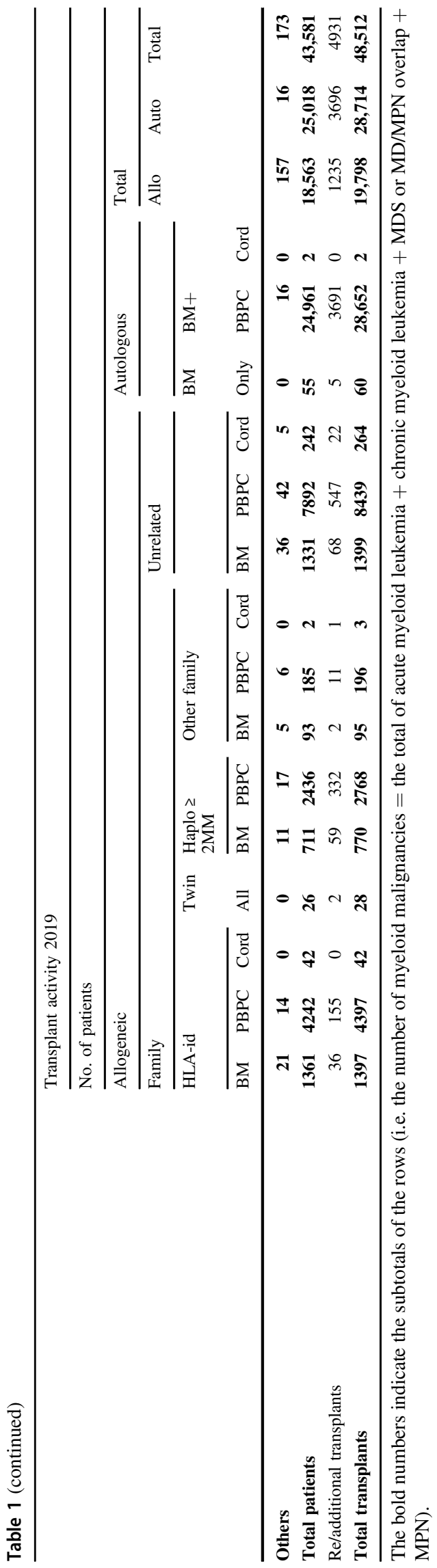

"A cell that has differentiated into a form capable of modulating or effecting a specific immune response" [25, 26]. This definition covers CAR-T cells and forms the basis for accreditation requirements in recent EBMT-JACIE recommendations [16].

Hematopoietic cellular therapies were categorized as chimeric antigen receptor T-cells (CAR -T); in vitro selected/and or expanded T-cells or cytokine activated, such as virus specific T-cells; cytokine-induced killer cells (CIK); regulatory $\mathrm{T}$ cells (TREGS); genetically modified $\mathrm{T}$ cells other than CAR-T; natural killer cells; dendritic cells; mesenchymal stromal cells; in vitro expanded CD34+ cells; and genetically modified CD34+ cells. This survey does not include cells from sources other than hematopoietic tissue [19]. On the other hand, gene therapy protocols, such as those used to treat thalassemia or SCID are part of this survey, but currently numbers are low.

\section{Transplant and cellular therapy rates}

Transplant rate, defined as the total number of HCT per 10 million inhabitants were computed for each country (based on the center report), without adjusting for patients receiving their HCT in a foreign country. Cellular therapy rates are defined as the numbers of patients receiving a cellular therapy treatment per 10 million population. Center density is defined as the number of centers performing a certain type of procedure per 10 million population. Population numbers for the European countries in 2019 were obtained from Eurostats: (http://appsso.eurostat.ec. europa.eu) and the World Bank database for the nonEuropean countries: (https://databank.worldbank.org).

\section{Analysis}

Wherever appropriate, the absolute numbers of transplanted patients, transplants or transplant rates are shown for specific countries, indications, or transplant techniques. Myeloid malignancy includes acute myeloid leukemia (AML), myelodysplastic or myelodysplastic/myeloproliferative neoplasia (MDS or MDS/MPN overlap), myeloproliferative neoplasm (MPN), and chronic myeloid leukemia (CML). Lymphoid malignancy includes acute lymphocytic leukemia (ALL), chronic lymphocytic leukemia (CLL), Hodgkin lymphoma (HL), non-Hodgkin lymphoma (NHL), and plasma cell disorders (PCD) (which includes multiple myeloma (MM) and others). The nonmalignant disorders include bone marrow failure (BMF (which includes severe aplastic anemia (SAA) and others), thalassemia and sickle cell disease (HG), primary immune disease (PID), inherited diseases of metabolism (IDM), and autoimmune diseases (AID). Others include histiocytosis and other rare disorders not included in the above. 


\section{Results}

\section{Participating centers in 2019}

Of the 700 centers, $451(64 \%)$ performed both allogeneic and autologous transplants; 229 (33\%) restricted their activity to autologous $\mathrm{HCT}$, and $18(3 \%)$ to allogeneic transplants only. Two of the 700 responding centers reported no activity due to renovation or changes within the transplant unit. Within the 698 actively transplanting centers in 2019, 128 (18\%) performed transplants on both adult and pediatric patients. An additional 122 (18\%) were dedicated pediatric transplant centers and 448 (64\%) perform transplants on adults only. Thirty centers failed to report in 2019, which, when compared with previously reported data, accounts for $\sim 780$ nonreported HCTs.

\section{Numbers of patients, transplants, and trends in 2019}

In 2019, 48,512 transplants were reported in 43,581 patients (first transplant); of these, 19,798 HCT (41\%) were allogeneic and 28,714 (59\%) autologous (Table 1). Compared with 2018, the total number of transplants increased by 2.2\% (0.9\% allogeneic HCT and 3.1\% autologous HCT) [13]. The corresponding number of patients showed an increase of $0.4 \%$ for allogeneic HCT and $2.5 \%$ for autologous HCT. In addition, there were $4931 \mathrm{~s}$ or subsequent transplants, 1235 being allogeneic, mainly to treat relapse or graft failure and 3696 autologous, the majority of which were likely to have been part of multiple transplant procedures such as tandem procedures, or as salvage autologous transplants for PCD. Furthermore, 819 of the allogeneic HCTs were reported as being given after a previous autologous HCT and were mainly for lymphoma or PCD.

The number of pediatric patients $(<18$ years old at transplant) transplanted in both dedicated pediatric and joint adult-pediatric units was 5189 (3990 allogeneic and 1199 autologous). This is an overall decrease of $3.3 \%$ in the total number of transplants; $2.1 \%$ allogeneic and $7.3 \%$ autologous HCT when compared to 2018. Of these, 3987 patients, (3123 allogeneic (78\%), and 864 autologous $(22 \%))$ were treated in 121 dedicated pediatric centers in 27 countries. Due to the design of the survey, detailed analysis is limited in the dedicated centers only. Main indications for allogeneic HCT were AML $(n=414 ; 70 \%$ in early stage), ALL ( $n=766 ; 45 \%$ in early stage), and NMD $(n=1534 ; 39 \%$ PID). There were 1625 family and 1498 unrelated donor HCTs reported. Within family donors, $42 \%$ were from a haploidentical relative. Bone marrow was used as the stem cell source in 1628 patients of which $56 \%$ were family donors. Peripheral blood stem cells were used in 1376 patients with equal proportions seen in both family $(n=685)$ and unrelated donors $(n=691)$. Cord blood stem cells were used in 119 patients of which $75 \%$ were unrelated HCT. The main indications for autologous HCT, were solid tumors, with 647 HCT reported in 2019, primarily for neuroblastoma $(49 \%)$.

\section{Main indications}

Indications for HCT in 2019 are listed in detail in Table 1 (Fig. 1a, b show distribution of disease indications for allogeneic (Fig. 1a) and autologous (Fig. 1b) HCT). Main indications for allogeneic HCT were myeloid malignancies (AML, CML, MDS or MDS/MPN overlap and MPN): 10,518 (98\% allogeneic HCT and 2\% autologous HCT). For autologous HCT, the main indications were lymphoid malignancies (ALL, CLL, PCD, HL, and NHL): 22,640 (19\% allogeneic HCT and $81 \%$ autologous HCT).

\section{Allogeneic HCT}

The leading indication for allogeneic HCT was AML, which accounts for $38 \%$ of all allogeneic HCT, an increase of $1 \%$ when compared to 2018. Increases were seen in both early disease stage $(1.7 \%)$ and therapy-related AML or those with myelodysplasia-related changes (3.5\%). AML in late disease stage decreased by $2.2 \%$. Among the myeloid malignancies, CML continues to increase overall by $6.2 \%$. However, differences were seen in 1 st chronic phase with a decrease of $14.4 \%$ and advanced phase which increased by $30.6 \%$, although overall the numbers remain low $(n=395$ patients). Allogeneic HCT for MDS decreased slightly by $0.5 \%$ to 2310 patients treated. MPN increased by $7.8 \%$ from 748 in 2018, to 806. ALL comprises $16.4 \%$ of allogeneic HCT and showed a slight increase overall of $2.7 \%$ compared to the previous year. Again, differences were seen between early stage, increasing by $4.8 \%$ and late stage, decreasing by $0.6 \%$. Allogeneic HCT for CLL continued to decrease by $10.9 \%$ when compared to 2018 , a constant trend over recent years. Allogeneic HCT for NHL decreased by $4.1 \%$ to 1310 while for $\mathrm{HL}$, rates remained stable. Within the nonmalignant disorders, a continued increase of $3.7 \%$ is seen for BMF-SAA $(n=749)$, and of $17.7 \%$ for BMF-non SAA $(n=273)$. PID increased by $6.4 \%(n=$ $719)$ and sickle cell disease by $44 \%(n=324)$. For IDM, the rate decreased by $11.6 \%(n=175)$ and for thalassemia by $17.4 \%(n=342)$. Allogeneic HCT for AID remain a rare indication with just 22 patients treated in 2019, although there is an overlap with PID and other inherited disorders that would benefit from better definition for future reporting [27]. Within allogeneic HCT, 7625 were performed using 


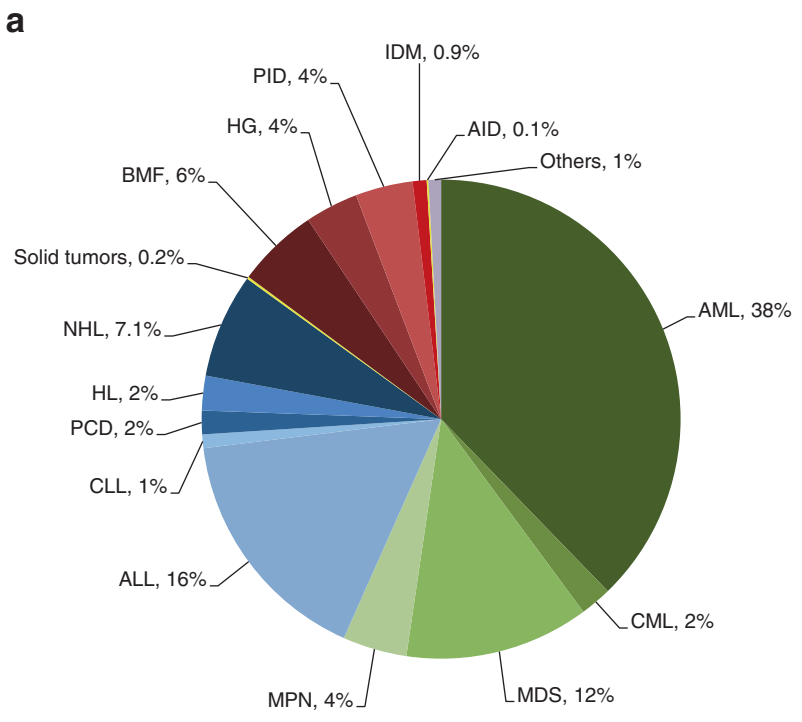

b

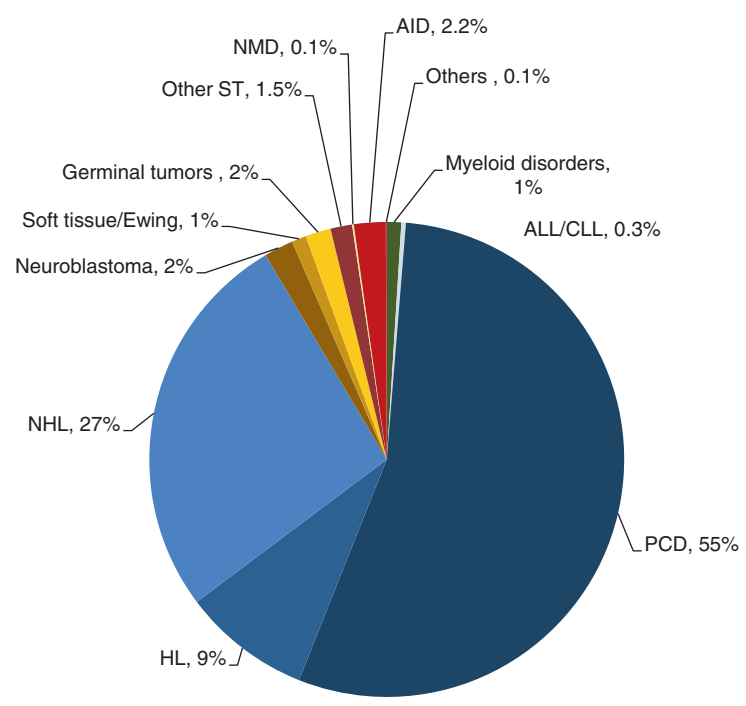

Fig. 1 Relative proportion of disease indications for HCT in Europe 2019. a Relative proportion of allogeneic HCT. b Relative proportion of autologous HCT.

non-myeloablative or reduced intensity conditioning in 2019. This comprises $39 \%$ of all allogeneic HCTs, a rate that has remained stable over the last 10 years.

\section{Autologous HCT}

The main indications for autologous HCT were lymphoid malignancies (90\%) with PCD comprising 55\% of all autologous HCT patients. Autologous HCT for NHL has not changed over time up to 2019 while PCD have increased slightly by $4.2 \%$. As reported in 2018, AML continued to decrease by $19.1 \%$ and ALL by $10.8 \%$. For solid tumors and AID, the numbers remained stable. In
2019, the activity survey was adapted to capture more defined data for AID. The majority were performed for multiple sclerosis $(n=442)$, followed by systemic sclerosis $(n=55)$ and other AID $(n=42)$.

\section{Transplant rates and center density}

Assessing transplant rates per 10 million population (TR) allows the comparison of activity in countries where population numbers differ greatly. Center density per 10 million population allows the comparison of activity by the number of centers. The TR rates for allogeneic HCT, ranged from 0.2 in Nigeria to 476.1 in Israel (median number of HCT 127 and TR 144) (Supplementary Fig. 1a). Five countries did not report any allogeneic HCT (Armenia, Bosnia and Herzegovina, Cyprus, Iceland, and Luxembourg). For autologous HCT, rates ranged from 2.3 in Syria to 625 in Switzerland (median number of HCT 181 and TR 281), (Supplementary Fig. 1b). Figure 2a shows the use of CAR-T cells in 2019 on a map as treatment rates and (Fig. 2b) as center density. Figure 2c, d correspondingly depict nonCAR-T cellular therapies as treatment rates and as center density respectively.

\section{Donor type and stem cell source}

In 2019, the overall numbers of patients treated with family donors remained stable, however, variation was seen within the choice of family donor used. HLA identical sibling and syngeneic twin donors decreased by $6 \%$ when compared to 2018 but increases were observed in haploidentical donors of $11 \%$ and unrelated donors of $1.2 \%$. The cord blood HCT rate continued to decrease slowly (1\%) and mainly included unrelated cord blood (85\%). In sibling donors, peripheral blood and bone marrow stem cell use decreased by $6.2 \%$ and $5.6 \%$ respectively. In haploidentical donors, an increase of $17.8 \%$ was seen in the use of stem cells harvested from peripheral blood while use of bone marrow stem cells decreased by $6.8 \%$. In unrelated donor transplants, the use of bone marrow and cord blood stem cells has decreased by $4.5 \%$ and $4 \%$, respectively. For allogeneic HCT, the numbers increased only slightly $(0.4 \%)$ and the proportion of those using bone marrow decreased by $5.4 \%$. When compared to 2018 , the absolute numbers of patients treated with autologous HCT has continued to increase (2.5\%) and since 1996 are predominantly performed using peripheral blood stem cells (>90\%).

\section{Thirty-year overview}

The first activity survey was conducted in 1990 and presented data on 4234 patients reported by 143 centers in 21 countries. 
a

Patients treated with CAR-T

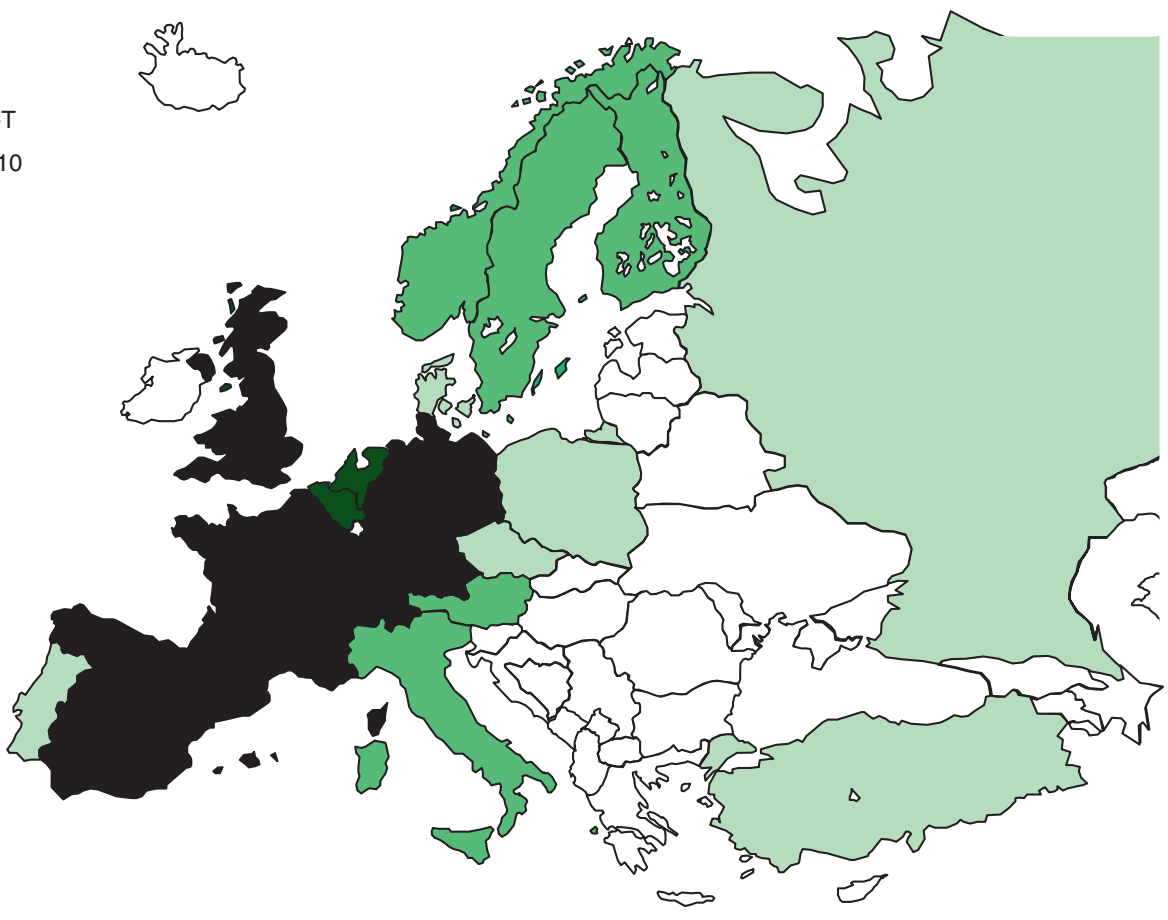

cellular therapy; rates per 10

million population

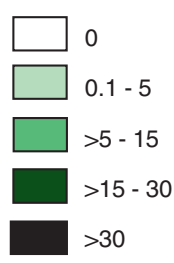

Israel

b

Number of centers performing

CAR-T cellular therapies per 10 million population

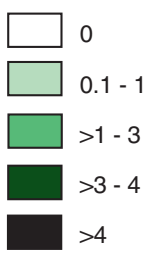

Israel

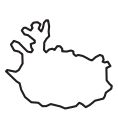


C

Patients treated with non CAR-T<smiles>C1CCCCCC1</smiles>
cellular therapy; rates per 10 million population

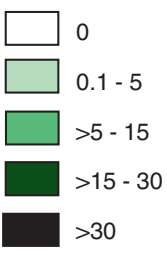

Iran, Lebanon Israel

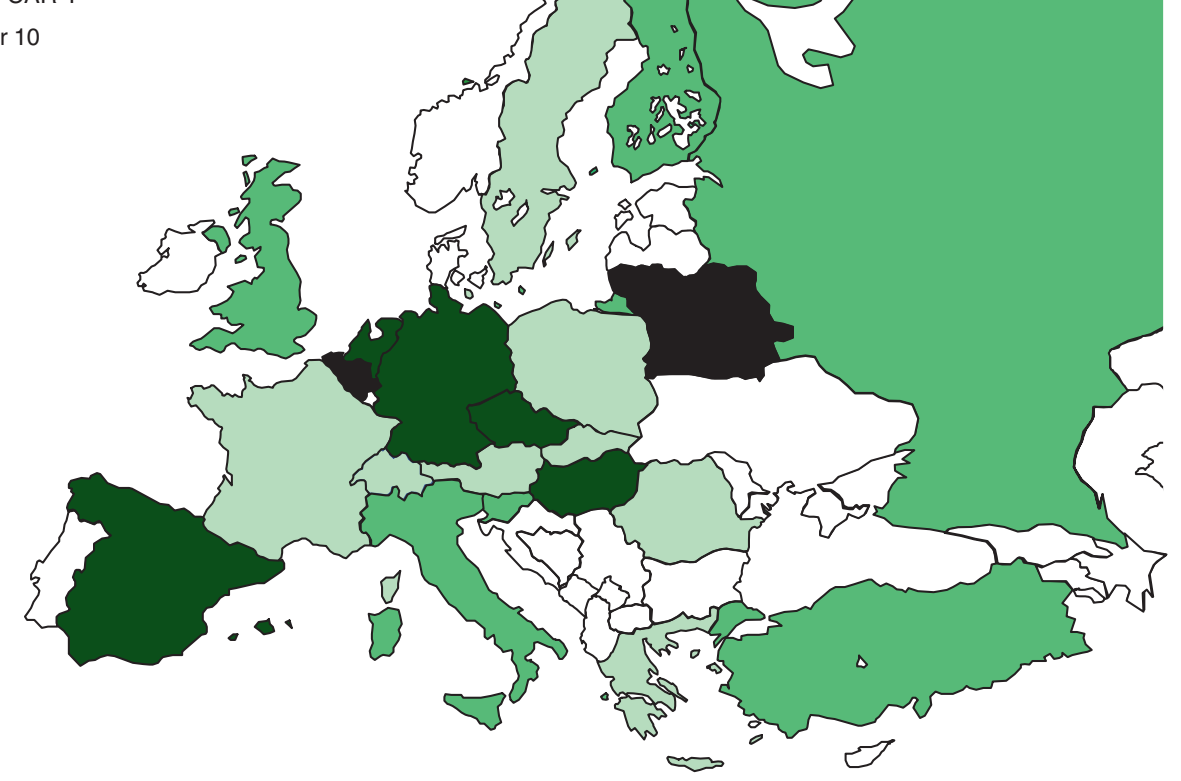

d

Number of centers performing non

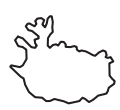

CAR-T cellular therapies per 10

million population

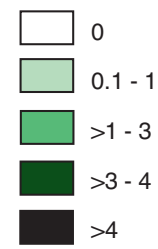

Iran

Lebanon, Israel

Fig. 2 Cellular therapy rates per 10 million population in Europe 2019. a Patients treated with CAR-T cellular therapy. b Number of centers performing CAR-T cellular therapies. $\mathbf{c}$ Patients treated with non CAR-T cellular therapy. d Number of centers performing non CAR-T cellular therapies. 
Fig. 3 Development of HCT from 1990 to 2019. a Number of patients receiving autologous and allogeneic HCT.

b Distribution of donor type among allogeneic HCT recipients. a

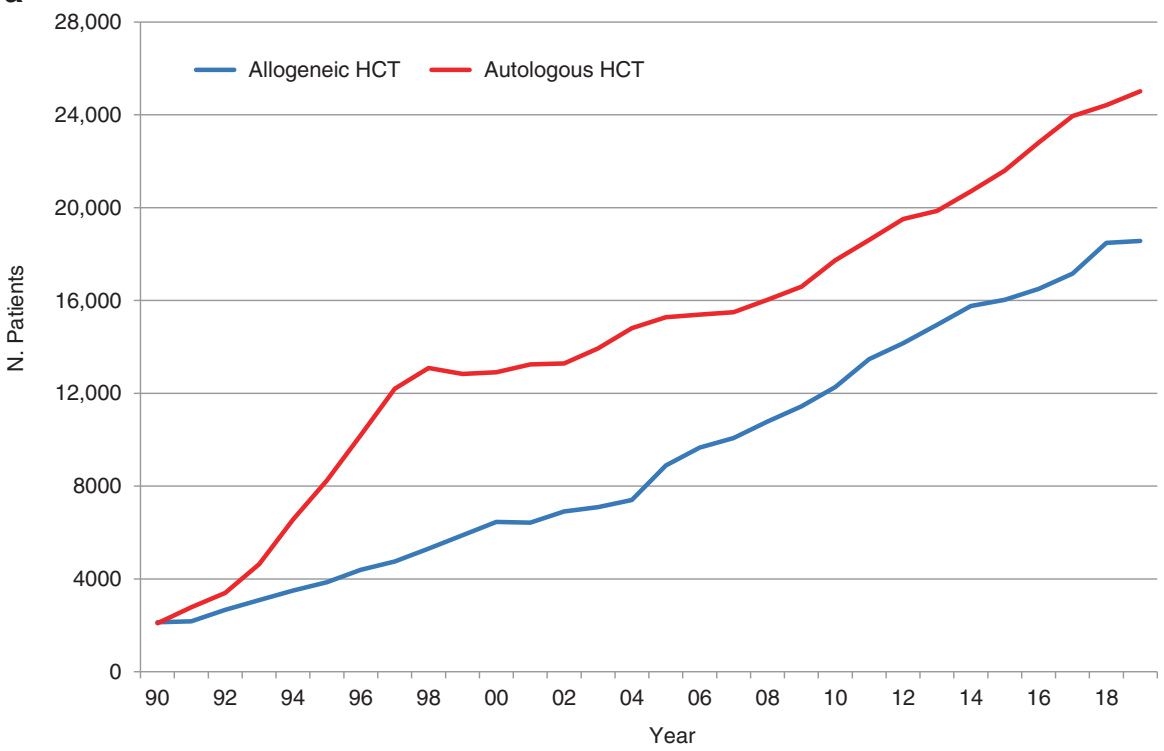

b

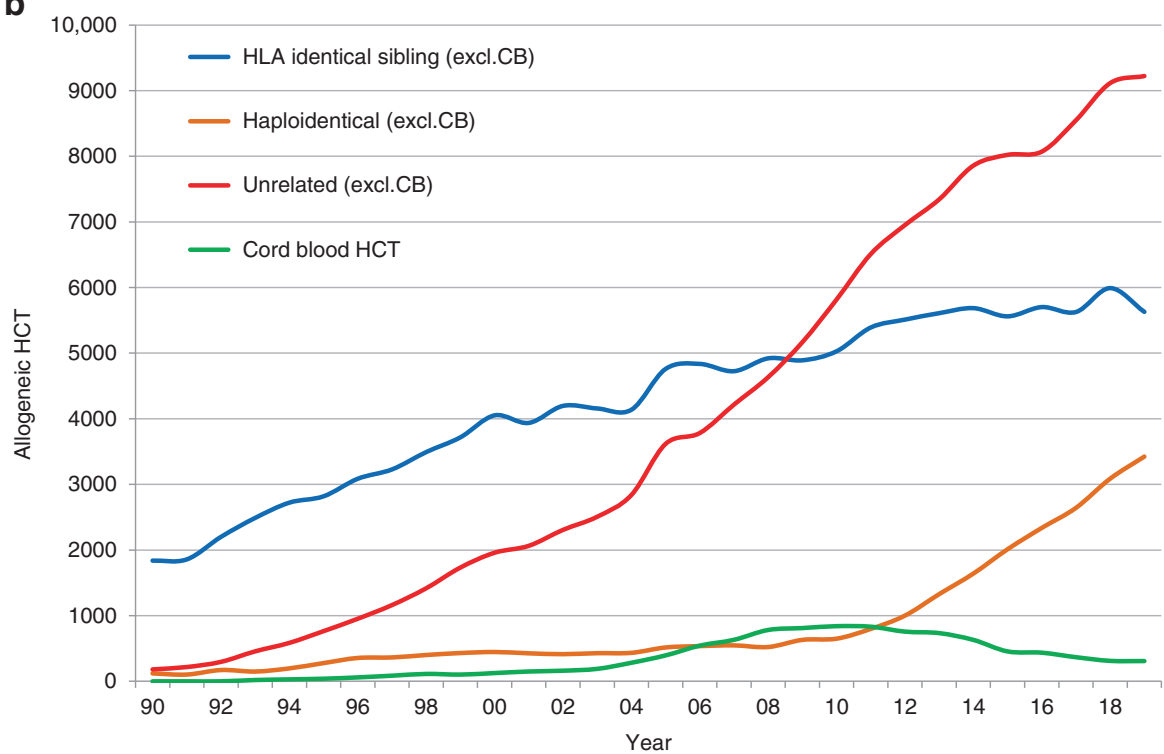

Since this first survey, revisions were made to ensure that each annual survey was optimized for the current changes in technology and treatment protocols used at the time. The survey allows us to monitor activities and trends over a 30 -year period. The number of reporting centers has increased from 143 to 700 in 2019, and from 20 countries to 51 . Allogeneic HCT has increased from 2137 to 19,798, while autologous HCT has increased from 2097 to 28,714.

Figure $3 \mathrm{a}$ shows the development in the numbers of patients treated with autologous and allogeneic HCT over the 30 -year period. Figure $3 \mathrm{~b}$ shows the distribution of donor type among allogeneic HCT recipients depicting the importance of unrelated donor and haploidentical donor HCT globally in the recent decade. Figure 4 shows the changes in choice of stem cell source for allogeneic HCT. Autologous HCT does not warrant graphical depiction as the use of bone marrow has almost disappeared. It is evident that for allogeneic HCT, growth is due to the use of peripheral blood stem cells; use of marrow remains stable at around 3500 transplants annually and is prevalent in allogeneic HCTs for nonmalignant disorders (Table 1). Figure 5 addresses the number of centers performing HCT in Europe, increasing from 143 to 700 over 30 years. The Figure shows the number of centers performing autologous and allogeneic HCT using sibling, unrelated and haploidentical donors as well as cord blood. The number of centers performing autologous HCT rose sharply in the late 90s, most likely associated with its use in the treatment of solid tumors. 
Fig. 4 Change in choice of stem cell source for allogeneic HCT from 1990 to 2019.

Figure shows the change in the use of bone marrow (BM), peripheral blood $(\mathrm{PB})$ and cord blood $(\mathrm{CB})$ as stem cell source over the 30 year period.
Fig. 5 Change in choice of donor type by center from 1990 to 2019. The figure shows the numbers of centers and the type of donors selected for HCT over the 30 year period.
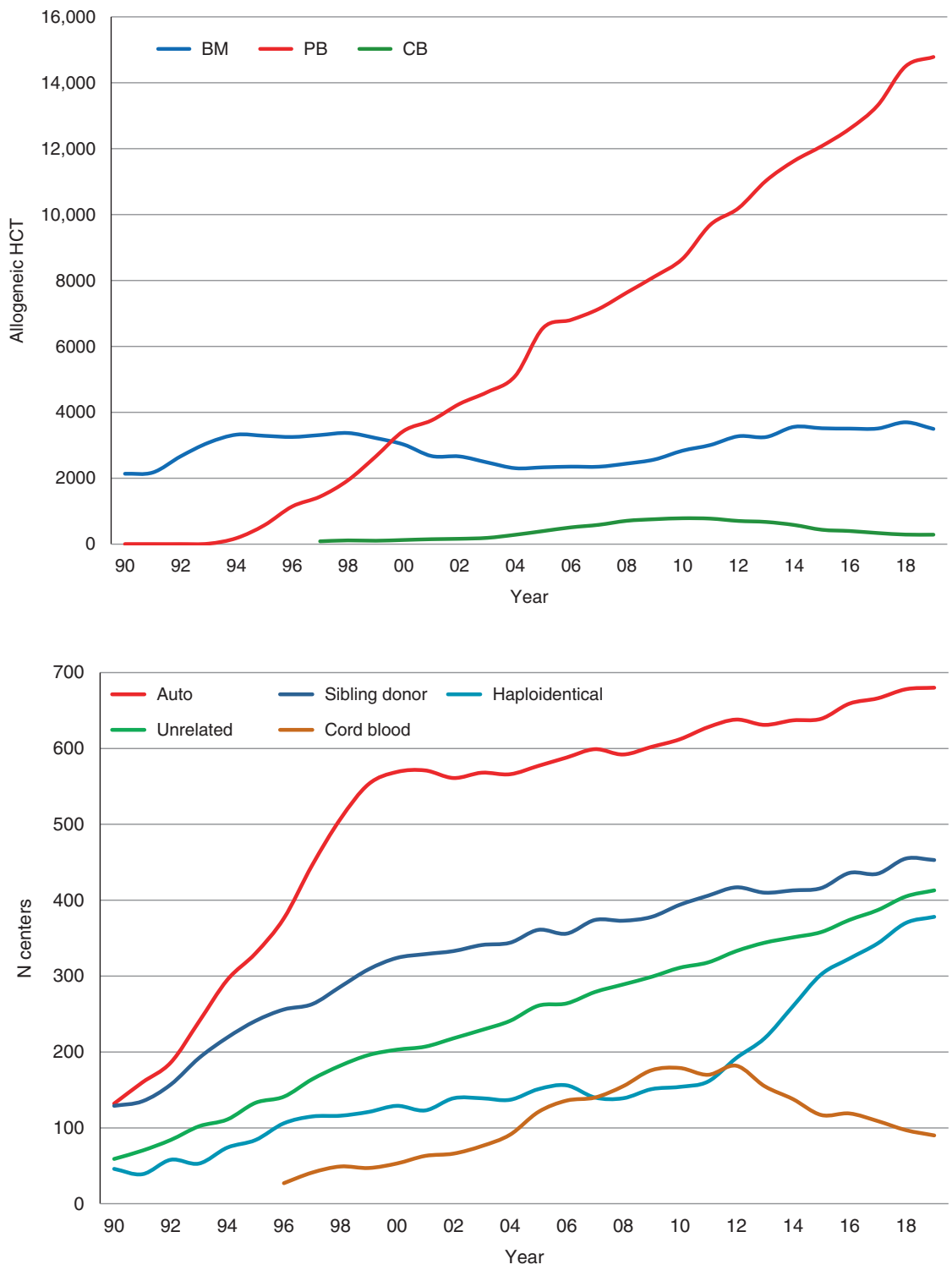

Regarding allogeneic HCT, at all points in time the number of centers performing transplants with donors other than HLA identical siblings was lower than the number of centers performing sibling transplants. Since around 2015, there appears to be a convergence of centers performing allogeneic HCT using all types of available donor. Since 2012 , the number of centers using cord blood donors as the stem cell source has declined.

\section{Cellular therapy}

Table 2 shows the number of patients receiving advanced cellular therapy and DLI performed in 2019. There were 3028 patients receiving unmanipulated DLIs, which is a slight decrease of $2.2 \%$ since 2018 . The majority of DLIs were given for relapse $(n=1461)$ and graft enhancement/ failure $(n=716)$.
A total of 2214 patients in 198 centers from 31 countries received other forms of hematopoietic cellular therapies that qualify as medicinal products rather than cell transplants [15]. In 2019 the most remarkable increase was in gene-modified $\mathrm{T}$ cells, notably CAR-T cells increasing from 151 in 2017 to 1134 in 2019 (650\% increase). One hundred and fifteen centers in 2019 in 19 countries reported CAR-T cellular therapies (Fig. 2a shows rates of CAR-T-cell treatment and Fig. $2 b$, center density performing CAR-T treatment). The main indication being lymphoma $(n=826 ; 100 \%$ autologous), followed by ALL ( $n=252 ; 92 \%$ autologous), and other malignancies ( $n=56$; $98 \%$ autologous). Much has been written about CAR-T cellular therapy replacing autologous or allogeneic HCT for lymphoma, Fig. 6 shows numbers of autologous (Fig. 6a) and allogeneic (Fig. 6b) HCT for NonHodgkin lymphoma and Hodgkin lymphoma over 30 years. At this point in time it is too early to predict whether 


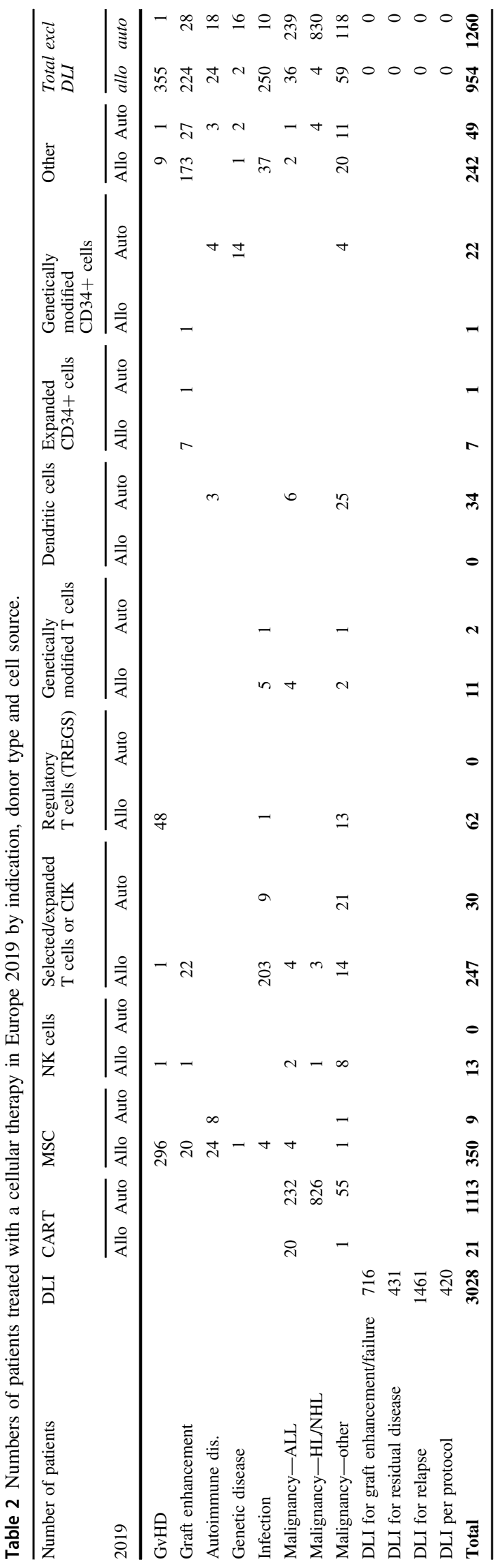

there has been an impact of CAR-T cell use on HCT. If the more than 800 CAR-T treatments for lymphoma had replaced HCT use, a notable decrease in activity would have been expected. The second most widely used cellular therapy other than CAR-T cells in 2019 is mesenchymal stromal cells $(n=$ 359 ; $97 \%$ allogeneic), their use being mainly to treat graftversus-host disease [19]. Since 2018, an increase was also seen in the numbers of selected/expanded $\mathrm{T}$ cells or CIK (127\%) and TREGS (59\%). Figure 2c shows rates of nonCAR-T-cell treatment and Fig. 2d, center density performing non-CAR-T treatment.

\section{Discussion}

The EBMT activity survey has been conducted annually since 1990 [1]. Over 48,000 transplants in more than 43,500 patients were reported in 2019. Starting in 2015, we observed a slower growth for unrelated donor HCT as compared to haploidentical HCT [12]. In the last year, however, use of both types of donor continues to increase; by $10 \%$ for haploidentical donors, and $1.9 \%$ for unrelated donors. It is mostly in well-established indications where growth is observed, such as allogeneic HCT for AML in CR1, but also ALL in CR1, MPN, and PID.

In the 2017 report we described lower rates of aplastic anemia transplantation possibly due to the use of thrombopoietin analogues such as eltrombopag. In the last 2 years though, we see the number has increased by $28.6 \%$, possibly suggesting that transplants are now performed later after failing thrombopoietin analogues but also centers proceeding quicker to transplantation as well as the growing upfront approach for children and younger adults.

The most impressive growth is observed in hematopoietic cellular therapies, most notably in the use of CAR-T cells, increasing to 1134 reported patients treated in 2019. Since the only two approved products received a centralized marketing approval from EMA in August 2018, it is possible that the reported activity in 2019 may still partially reflect patients in clinical studies, either industry-sponsored or academia sponsored [https://www.ema.europa.eu/en/ documents/scientific-guideline/qualification-opinion-cellula r-therapy-module-european-society-blood-marrow-transpla ntation-ebmt_en.pdf]. Investigational CAR-T cells can be produced by academic facilities. Since the EMA approved the first CAR-T cell product, a notable increase in the use of CAR-T cells has been observed and a further increase is to be expected in 2020 [22, 23]. Emergence of a rapidly growing clinical activity is reassuring in view of earlier reports demonstrating that Europe lagged behind the USA and China [24]. Figure 2b illustrates wide variability in center density for CAR-T cell treatment. Obviously, it is difficult to state an optimal number of centers for a given 
Fig. 6 Change in HCT for lymphomas 1990-2019.

a Autologous HCT for Hodgkin and non-Hodgkin lymphoma. b Allogeneic HCT for Hodgkin and non-Hodgkin lymphoma.

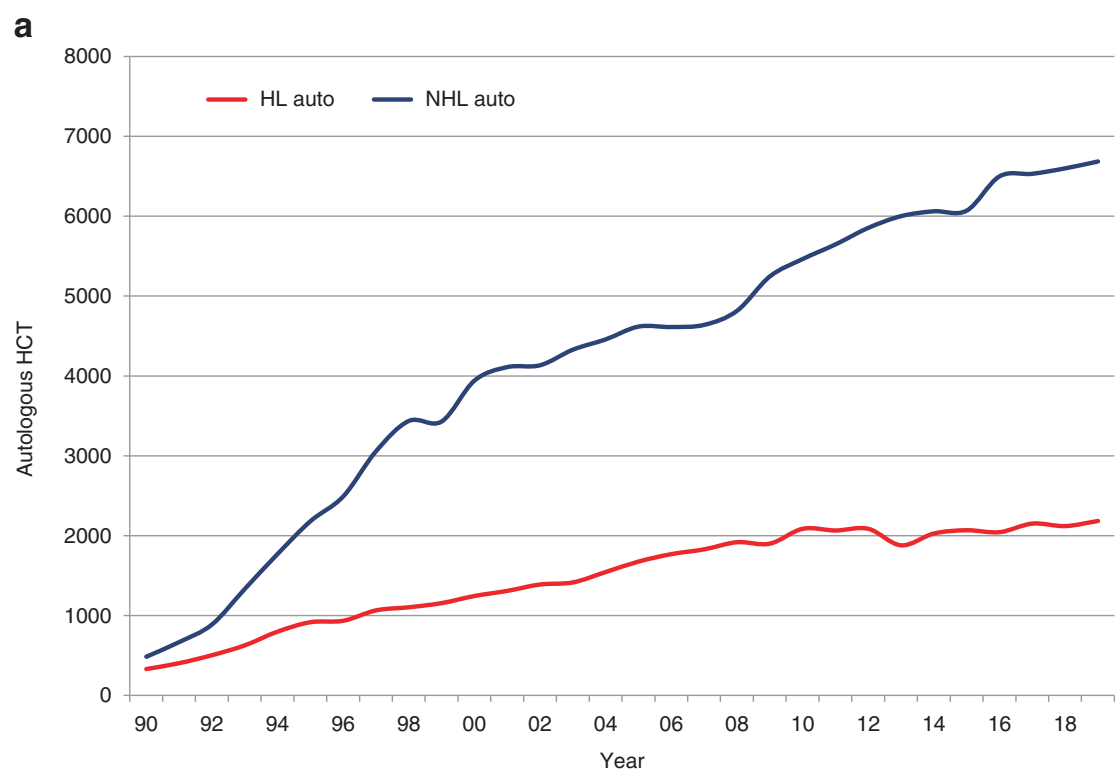

b

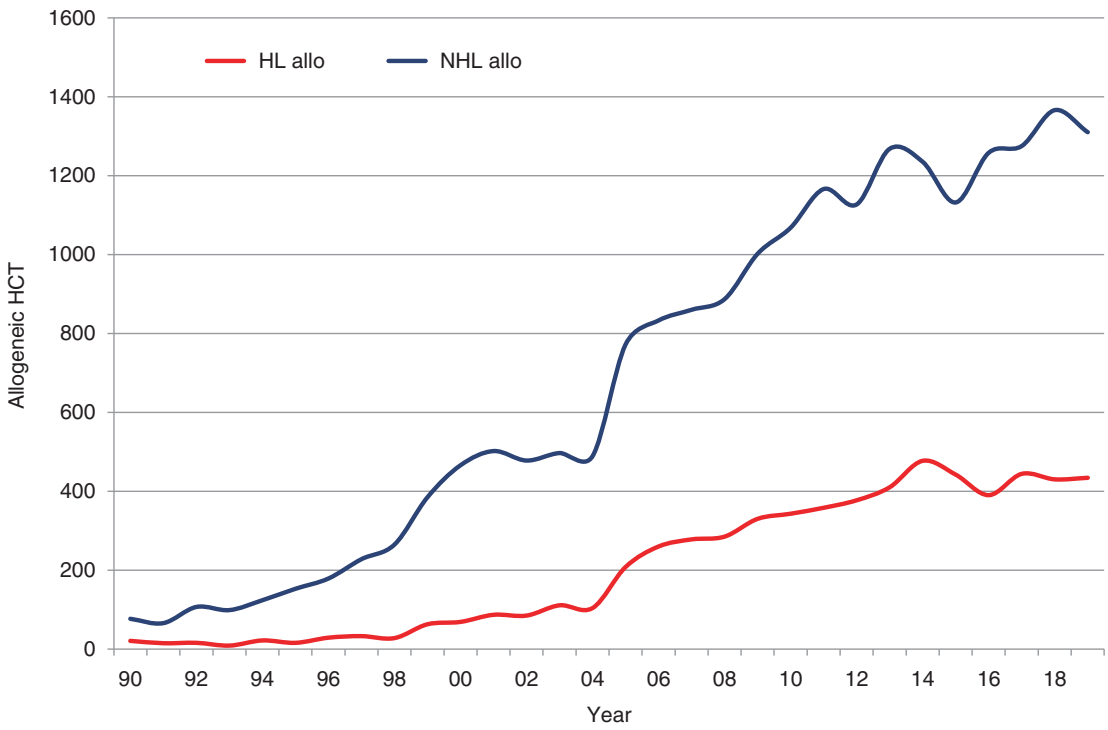

population at this point in time. The survey will continue to observe this development. The constant and steady increase in autologous or allogeneic HCT for NHL has not changed over time. These are indications for autologous as well as for allogeneic HCT which may in the future be replaced by CAR-T treatments. So far, there is little evidence for a replacement of transplant technology by cellular therapy products. While the use of transplant technology for autologous HCT for PCD continues to increase, the use of allogeneic HCT for PCD is decreasing; this may be due to the availability of more potent drugs such as monoclonal antibodies. The 30-year comparison shows the impressive expansion of the use of HCT technology across Europe. Whereas one can still criticize a lack of standardization in transplant indications and technologies, continued growth in established indications hints at a convergence of practices across Europe. Of interest is the use of HCT using alternative donors in Europe where centers specializing in certain types of transplants have given way to centers more commonly using the entire spectrum of sibling, unrelated donor and haploidentical donor transplantation reflecting an increased choice of donor available for patients in need of allogeneic HCT.

The annual activity survey of the EBMT reflects current activity and trends in the field of transplant technology. It is valuable for the dissemination of the most recent information on indications, donor and stem cell usage, and benchmarking of data completeness and survival outcomes [28], which will ultimately be beneficial in health care planning. 
Acknowledgements Special thanks go to the cooperation of all participating centers and their staff (listed in the Supplementary Online Appendix). The staff at the EBMT Co-ordination offices; Barcelona, Paris and London. The National Registries; Austria (ASCTR), Belgium, Czech Republic, France (SFGM-TC), Germany (DRST) Italy (GITMO), The Netherlands, Spain (GETH), Switzerland (SBST), and the United Kingdom (BSBMT). The authors also thank D. John for database support.

Funding Open Access funding provided by Lib4RI - Library for the Research Institutes within the ETH Domain: Eawag, Empa, PSI \& WSL.

\section{Compliance with ethical standards}

Conflict of interest The authors declare no competing interests. The writing of the paper was the sole responsibility of the authors.

Publisher's note Springer Nature remains neutral with regard to jurisdictional claims in published maps and institutional affiliations.

Open Access This article is licensed under a Creative Commons Attribution 4.0 International License, which permits use, sharing, adaptation, distribution and reproduction in any medium or format, as long as you give appropriate credit to the original author(s) and the source, provide a link to the Creative Commons license, and indicate if changes were made. The images or other third party material in this article are included in the article's Creative Commons license, unless indicated otherwise in a credit line to the material. If material is not included in the article's Creative Commons license and your intended use is not permitted by statutory regulation or exceeds the permitted use, you will need to obtain permission directly from the copyright holder. To view a copy of this license, visit http://creativecommons. org/licenses/by/4.0/.

\section{References}

1. Gratwohl A. Bone marrow transplantation activity in Europe 1990. Report from the European Group for Bone Marrow Transplantation (EBMT). Bone Marrow Transplant. 1991;8:197-201

2. Copelan EA, Chojecki A, Lazarus HM, Avalos BR. Allogeneic hematopoietic cell transplantation; the current renaissance. Blood Rev. 2019;34:34-44.

3. Appelbaum FR. Hematopoietic-cell transplantation at 50. N Engl J Med. 2007;357:1472-5.

4. Duarte RF, Labopin M, Bader P, Basak GW, Bonini C, Chabannon $\mathrm{C}$, et al. Indications for haematopoietic stem cell transplantation for haematological diseases, solid tumours and immune disorders: current practice in Europe, 2019. Bone Marrow Transplant. 2019;54:1525-52.

5. Gratwohl A, Baldomero H, Aljurf M, Pasquini MC, Bouzas LF, Yoshimi A, et al. Hematopoietic stem cell transplantation: a global perspective. JAMA. 2010;303:1617-24.

6. Gratwohl A, Pasquini MC, Aljurf M, Atsuta Y, Baldomero H, Foeken L, et al. One million haemopoietic stem-cell transplants: a retrospective observational study. Lancet Haematol. 2015;2:e91-100.

7. Niederwieser D, Baldomero H, Szer J, Gratwohl M, Aljurf M, Atsuta Y, et al. Hematopoietic stem cell transplantation activity worldwide in 2012 and a SWOT analysis of the Worldwide Network for Blood and Marrow Transplantation Group including the global survey. Bone Marrow Transplant. 2016;51:778-85.

8. Gratwohl A, Schwendener A, Baldomero H, Gratwohl M, Apperley J, Niederwieser D, et al. Changes in use of hematopoietic stem cell transplantation; a model for diffusion of medical technology. Haematologica. 2010;95:637-43.

9. Passweg JR, Baldomero H, Bader P, Bonini C, Cesaro S, Dreger $\mathrm{P}$, et al. Hematopoietic stem cell transplantation in Europe 2014: more than 40000 transplants annually. Bone Marrow Transplant. 2016;51:786-92.

10. Passweg JR, Baldomero H, Bader P, Bonini C, Duarte RF, Dufour $\mathrm{C}$, et al. Use of haploidentical stem cell transplantation continues to increase; the 2015 European Society for Blood and Marrow Transplant activity survey report. Bone Marrow Transplant. 2017;52:811-7.

11. Majhail NS, Mau LW, Chitphakdithai P, Denzen EM, Joffe S, Lee $\mathrm{SJ}$, et al. Transplant center characteristics and survival after allogeneic hematopoietic cell transplantation in adults. Bone Marrow Transplant. 2020;55:906-17.

12. Passweg JR, Baldomero H, Bader P, Basak GW, Bonini C, Duarte $\mathrm{R}$, et al. Is the use of unrelated donor transplantation leveling off in Europe? The 2016 European Society for Blood and Marrow Transplant activity survey report. Bone Marrow Transplant. 2018;53:1139-48.

13. Passweg JR, Baldomero H, Chabannon C, Basak GW, Corbacioglu S, Duarte R, et al. The EBMT activity survey on hematopoietic-cell transplantation and cellular therapy 2018: CART's come into focus. Bone Marrow Transplant. 2020;55:1604-13.

14. Shouval R, Fein JA, Labopin M, Kröger N, Duarte RF, Bader P, et al. Outcomes of allogeneic haematopoietic stem cell transplantation from HLA-matched and alternative donors: a European Society for Blood and Marrow Transplantation registry retrospective analysis. Lancet Haematol. 2019;6:e573-84.

15. Chabannon C, Hildebrandt M, Scheding S, Humpe A, Lowdell M, Slaper-Cortenbach I. Regulation of advanced therapy medicinal products will affect the practice of haematopoietic SCT in the near future: a perspective from the EBMT cell-processing committee. Bone Marrow Transplant. 2015;50:321-3.

16. Yakoub-Agha I, Chabannon C, Bader P, Basak GW, Bonig H, Ciceri $\mathrm{F}$, et al. Management of adults and children undergoing CAR t-cell therapy: best practice recommendations of the European Society for Blood and Marrow Transplantation (EBMT) and the Joint Accreditation Committee of ISCT and EBMT (JACIE). Haematologica. 2020;105:297-316.

17. Bonini C, Mondino A. Adoptive T-cell therapy for cancer: the era of engineered T cells. Eur J Immunol. 2015;45:2457-69.

18. Chabannon C, Kuball J, Bondanza A, Dazzi F, Pedrazzoli P, Toubert A, et al. Hematopoietic stem cell transplantation in its 60s: A platform for cellular therapies. Sci Transl Med. 2018;10: eaap9630.

19. Tolar J, Le Blanc K, Keating A, Blazar BR. Concise review: hitting the right spot with mesenchymal stromal cells. Stem Cells. 2010;28:1446-55.

20. June $\mathrm{CH}$, Sadelain M. Chimeric antigen receptor therapy. N Engl J Med. 2018;379:64-73.

21. Maude SL, Frey N, Shaw PA, Aplenc R, Barrett DM, Bunin NJ, et al. Chimeric antigen receptor $\mathrm{T}$ cells for sustained remissions in leukemia. N Engl J Med. 2014;371:1507-17.

22. Schuster SJ, Bishop MR, Tam CS, Waller EK, Borchmann P, McGuirk JP, et al. Tisagenlecleucel in Adult Relapsed or Refractory Diffuse Large B-Cell Lymphoma. N Engl J Med. 2019;380:45-56.

23. Shah NN, Fry TJ. Mechanisms of resistance to CAR $T$ cell therapy. Nat Rev Clin Oncol. 2019;16:372-85.

24. Hartmann J, Schüßler-Lenz M, Bondanza A, Buchholz CJ. Clinical development of CAR T cells-challenges and opportunities in translating innovative treatment concepts. EMBO Mol Med. 2017;9:1183-97.

25. Snowden JA, McGrath E, Duarte RF, Saccardi R, Orchard K, Worel $\mathrm{N}$, et al. JACIE accreditation for blood and marrow 
transplantation: past, present and future directions of an international model for healthcare quality improvement. Bone Marrow Transplant. 2017;52:1367-71.

26. Saccardi R, McGrath E, Snowden JA. JACIE accreditation of HSCT programs. In: Carreras E, Dufour C, Mohty M, Kröger N, editors. The EBMT Handbook. Cham: Springer; 2019. p. $35-40$

27. Greco R, Labopin M, Badoglio M, Veys P, Furtado Silva JM, Abinun M, et. al. Allogeneic HSCT for autoimmune diseases: a retrospective study from the EBMT ADWP, IEWP, and PDWP working parties. Front Immunol. 2019:10:1570.

28. Snowden JA, Saccardi R, Orchard K, Ljungman P, Duarte RF, Labopin $\mathrm{M}$, et al. Benchmarking of survival outcomes following haematopoietic stem cell transplantation: a review of existing processes and the introduction of an international system from the European Society for Blood and Marrow Transplantation (EBMT) and the Joint Accreditation Committee of ISCT and EBMT (JACIE). Bone Marrow Transplant. 2020;55:681-94.

\section{Affiliations}

Jakob R. Passweg ${ }^{1} \cdot$ Helen Baldomero $\mathbb{1}^{1} \cdot$ Christian Chabannon $\mathbb{1}^{2} \cdot$ Grzegorz W. Basak $\mathbb{1}^{3} \cdot$ Rafael de la Cámara $\mathbb{1}^{4} \cdot$ Selim Corbacioglu ${ }^{5} \cdot$ Harry Dolstra $^{6} \cdot$ Rafael Duarte $^{7}$ - Bertram Glass ${ }^{8} \cdot$ Raffaella Greco $\mathbb{1}^{9}$. Arjan C. Lankester ${ }^{10} \cdot$ Mohamad Mohty $^{11} \cdot$ Régis Peffault de Latour ${ }^{12} \cdot$ John A. Snowden $^{13}$. Ibrahim Yakoub-Agha ${ }^{14}{ }^{14}$. Nicolaus Kröger ${ }^{15}$. for the European Society for Blood and Marrow Transplantation (EBMT)

1 EBMT Activity Survey Office, Division of Hematology, Department of Medicine, University Hospital, Basel, Switzerland

2 Institut Paoli Calmettes Comprehensive Cancer Center \& Inserm CBT-1409, Centre d'Investigations Cliniques en Biothérapies, Marseille, France

3 Department of Hematology, Oncology and Internal Medicine, Medical University of Warsaw, Warsaw, Poland

4 Department of Hematology, Hospital Universitario de la Princesa, Madrid, Spain

5 Department of Pediatric Hematology, Oncology and Stem Cell Transplantation, University of Regensburg, Regensburg, Germany

6 Laboratory of Hematology, Department of Laboratory Medicine, Radboud University Medical Center, Nijmegen, The Netherlands

7 Servicio de Hematologia y Hemoterapia, Hospital Universitario Puerta de Hierro, Madrid, Spain

8 Klinik für Hämatologie und Stammzelltransplantation, HELIOS Klinikum Berlin-Buch, Berlin, Germany
9 Unit of Hematology and Bone Marrow Transplantation, IRCCS San Raffaele Scientific Institute, Vita-Salute San Raffaele University, Milan, Italy

10 Willem-Alexander Children's Hospital, Department of Pediatrics, Leiden University Medical Centre Leiden, Leiden, The Netherlands

11 Department of Hematology, Hospital Saint Antoine, INSERM UMRs938, Sorbonne University, Paris, France

12 BMT Unit, Department of Hematology, Hospital St. Louis, Paris, France

13 Department of Haematology, Sheffield Teaching Hospitals NHS Foundation Trust, Sheffield, UK

14 CHU de Lille, INSERM U1286, Infinite, Univ Lille, Lille, France

15 Department of Stem Cell Transplantation, University Hospital Eppendorf, Hamburg, Germany 